\title{
Observation of Mixed Fermionic-Bosonic Helium Clusters by Transmission Grating Diffraction
}

\author{
Anton Kalinin, Oleg Kornilov, ${ }^{*}$ Wieland Schöllkopf, ${ }^{\dagger}$ and J. Peter Toennies \\ Max-Planck-Institut für Dynamik und Selbstorganisation, Bunsenstraße 10, D-37073 Göttingen, Germany
}

(Received 7 June 2005; published 9 September 2005)

\begin{abstract}
Small weakly bound boson-fermion ${ }^{4} \mathrm{He}_{m}{ }^{3} \mathrm{He}_{n}$ clusters formed in a free jet expansion are identified using nondestructive transmission grating diffraction. The observations confirm the existence of more than 11 very tenuous complexes including the three-body halo molecule ${ }^{4} \mathrm{He}_{2}{ }^{3} \mathrm{He}$ and the pseudo-Borromean complex ${ }^{4} \mathrm{He}_{2}{ }^{3} \mathrm{He}_{2}$. Effective cluster formation temperatures, extracted from a sudden freeze model for cluster growth using theoretical binding energies, increase smoothly with cluster size, thereby confirming the calculations with the possible exception of ${ }^{4} \mathrm{He}_{2}{ }^{3} \mathrm{He}_{2}$.
\end{abstract}

DOI: $10.1103 /$ PhysRevLett.95.113402

PACS numbers: $36.40 . Q v, 21.45 .+\mathrm{v}, 61.46 .+\mathrm{w}$

All matter consists of either bosons or fermions and obeys different symmetries on exchange of particles. Whereas the properties of most condensed solids or liquids do not depend significantly on the particle symmetry, in the case of helium there are dramatic differences between bosonic ${ }^{4} \mathrm{He}$ and fermionic ${ }^{3} \mathrm{He}$. ${ }^{4} \mathrm{He}$ is a superfluid at temperatures below $2.2 \mathrm{~K}$, whereas in ${ }^{3} \mathrm{He}$ superfluidity occurs only below about $3 \times 10^{-3} \mathrm{~K}$ when Cooper pairs can be stabilized. The many recent theoretical studies of small helium clusters provide a new bottom-up approach for understanding these fascinating differences [1]. In the comparatively few experiments reported so far, molecular beams of small pure ${ }^{4} \mathrm{He}$ clusters from the very weakly bound dimer $\left(E_{b}=1.3 \times 10^{-3} \mathrm{~K}\right)$ [2] upwards to clusters with about 100 atoms [3] have been studied by diffraction from a nanostructured transmission grating. Although pure fermionic ${ }^{3} \mathrm{He}$ clusters are ideal models for understanding the role of Fermi symmetry in nuclei and, like nuclei, are expected to exhibit shell closure [4], up to now they could not be studied experimentally. Their greater zero point energy and the Pauli exclusion principle prevent less than about $32{ }^{3} \mathrm{He}$ atoms from forming a stable cluster [5]. Thus in free jet expansions they condense abruptly to produce clusters too large to be resolved with matter-wave diffraction.

Small mixed clusters ${ }^{4} \mathrm{He}_{m}{ }^{3} \mathrm{He}_{n}$, denoted in the following as $(m, n)$ clusters, are, however, predicted to be stable. They also exhibit fermionic shell closure and have since 1979 attracted considerable attention from theoreticians [6-11]. Although the zero point energy is too large for the mixed ${ }^{4} \mathrm{He}^{3} \mathrm{He}$ dimer to be bound, the ${ }^{4} \mathrm{He}_{2}{ }^{3} \mathrm{He}$ trimer is just barely stable with a binding energy of only $0.0142 \mathrm{~K}$ [10] and exhibits a pronounced three-body halo with many similarities to the first excited Efimov state in ${ }^{4} \mathrm{He}_{3}$ [7]. Since two of its three pairs are unbound, it can be classified as an example of a tango state [11]. The ${ }^{4} \mathrm{He}_{2}{ }^{3} \mathrm{He}_{2}$ complex is even more interesting. Although only one of its six pairs is bound, making it a pseudo-Borromean system, it has a single state weakly bound by $0.100 \mathrm{~K}[8,10]$, nearly 6 times less than the binding energy of $0.560 \mathrm{~K}$ for the pure bosonic
${ }^{4} \mathrm{He}$ tetramer [12]. With additional ${ }^{3} \mathrm{He}$ atoms the clusters with $2{ }^{4} \mathrm{He}$ atoms become unstable, but then are stable once again with $8{ }^{3} \mathrm{He}$ atoms [9]. The ${ }^{4} \mathrm{He}_{4}{ }^{3} \mathrm{He}_{2}$ cluster appears to be the smallest cluster, which has an excited $(L, S)=$ $(1,1)$ state, reminiscent of the spin-triplet $p$-state Cooper pairs in superfluid liquid ${ }^{3} \mathrm{He}$.

In the present experiments mixed clusters are formed in a cryogenic free jet expansion of a mixture of the ${ }^{4} \mathrm{He}$ and ${ }^{3} \mathrm{He}$ gases and analyzed for their masses nondestructively with the matter-wave diffraction technique used previously for small ${ }^{4} \mathrm{He}$ clusters [2,3]. Compared to the previous experiments the cluster intensities are lower by 1 to 2 orders of magnitude, since the concentration of ${ }^{4} \mathrm{He}$ had to be reduced to a few percent in order to suppress the formation of the more stable pure ${ }^{4} \mathrm{He}$ clusters. Despite the low signals of typically less than 1 count/s the existence of both the ${ }^{4} \mathrm{He}_{2}{ }^{3} \mathrm{He}$ and ${ }^{4} \mathrm{He}_{2}{ }^{3} \mathrm{He}_{2}$ as well as 9 other larger clusters including ${ }^{4} \mathrm{He}_{4}{ }^{3} \mathrm{He}_{3}$ could be confirmed. Furthermore, an analysis of the relative intensities based on a sudden freeze growth model provides qualitative confirmation of the theoretically predicted binding energies with the possible exception of ${ }^{4} \mathrm{He}_{2}{ }^{3} \mathrm{He}_{2}$.

The measurements were carried out on an apparatus described in detail in Ref. [13]. The mixture of the commercially purified gases is expanded through a 5 - $\mu \mathrm{m}$-diameter orifice at source temperatures $T_{0}$ between 3.1 and $6.7 \mathrm{~K}$ and pressures $P_{0}$ between 0.75 and $4.5 \mathrm{bar}$. After collimation by two $5 \mathrm{~mm}$ high $20 \mu \mathrm{m}$ wide slits located at 23 and $105 \mathrm{~cm}$ downstream from the source, the particles are diffracted by a $d \cong 100 \mathrm{~nm}$ period $\mathrm{SiN}_{x}$ transmission grating [13] located at $115 \mathrm{~cm}$ from the source. A slit in front of the electron-impact-ionization mass spectrometer detector, located at $140 \mathrm{~cm}$ from the grating, provides for an angular resolution of $\Delta \vartheta \cong 70 \mu \mathrm{rad}$. The cluster ion fragments are measured at either 3 amu (pure ${ }^{3} \mathrm{He}$ and mixed clusters), 4 and $8 \mathrm{amu}$ (pure ${ }^{4} \mathrm{He}$ and mixed clusters), or $7 \mathrm{amu}$ (only mixed clusters). Since all the clusters have the same velocities $v$ to within about $1 \%[3,14]$, the sharp first order diffraction peaks appear at angles $\vartheta \cong$ $\lambda / d=h(M v d)^{-1}$, which are inversely proportional to the 
integral mass $M$ of the clusters ( $\lambda$ and $h$ denote the wavelength and the Planck's constant, respectively).

Figure 1 provides an overview of the diffraction patterns obtained using $9 \%-10 \%$ rich ${ }^{4} \mathrm{He} /{ }^{3} \mathrm{He}$ mixtures with the mass spectrometer set at mass $3 \mathrm{amu}$ (a), 4 amu (b), and $7 \mathrm{amu}$ (c),(d). Only first order peaks on one side of the symmetric patterns are shown. The diffraction pattern on mass 3 amu [Fig. 1(a)] exhibits only a single peak at about $-5.7 \mathrm{mrad}$, which is attributed to ${ }^{3} \mathrm{He}$ atoms. The ${ }^{4} \mathrm{He}$ diffraction pattern in (b) measured on mass 4 amu exhibits, in addition to an atom peak at $-4.3 \mathrm{mrad}$, a number of peaks at smaller angles, corresponding to ${ }^{4} \mathrm{He}_{n}$ clusters identified in (b), as reported earlier for pure ${ }^{4} \mathrm{He}$ cluster beams [14].

The mixed clusters stand out more clearly on mass 7 amu in (c) and (d) plotted on an expanded angular scale. Distinct structures can be detected above the background of 0.5 counts/s after measuring times of up to $20-30 \mathrm{~h}$. In assigning the peaks the signal was fitted with Gaussians of equal width centered at the integer-mass angular positions interpolated from the clearly resolved peaks. According to the bottom abscissa the following mixed clusters can be identified: $(m, n)=(2,1),(3,1),(4,1)$, an unresolved peak

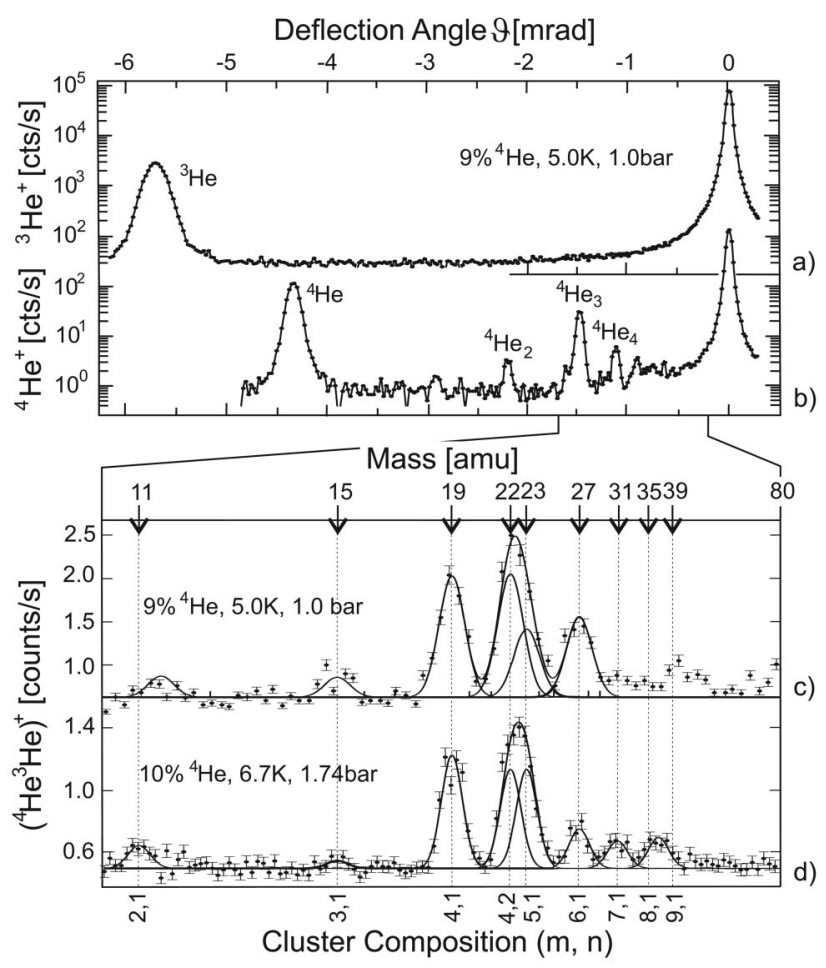

FIG. 1. Diffraction patterns of clusters in a cryogenic free jet expansion of $9 \%-10 \%{ }^{4} \mathrm{He}$ in ${ }^{3} \mathrm{He}$ gas mixtures measured for different ion fragments: (a) on mass $3 \mathrm{amu}\left({ }^{3} \mathrm{He}^{+}\right)$, (b) on mass $4 \mathrm{amu}\left({ }^{4} \mathrm{He}^{+}\right)$, and (c),(d) on mass $7 \mathrm{amu}\left({ }^{4} \mathrm{He}^{3} \mathrm{He}^{+}\right)$, which are assigned to mixed clusters ${ }^{4} \mathrm{He}_{m}{ }^{3} \mathrm{He}_{n}$. The diffraction angles in (d) correspond to the same masses after correcting for the different beam velocity compared to (c). The top and bottom abscissas in (c) and (d) show the assigned cluster masses and compositions, respectively. consisting of $(4,2)$ and $(5,1)$, peaks centered at $(6,1)$ and $(7,1)$, and a broad peak centered at about $M=37 \mathrm{amu}$. The lack of evidence for mixed clusters with more than 1 ${ }^{3} \mathrm{He}$ atom, with the possible exception of the $(4,2)$ cluster, is attributed to the competing formation of the more stable pure ${ }^{4} \mathrm{He}$ clusters. To compensate for this the ${ }^{4} \mathrm{He}$ concentration was reduced to $1 \%$. Figure 2 compares a diffraction pattern on $8 \mathrm{amu}\left({ }^{4} \mathrm{He}_{2}{ }^{+}\right)$[Fig. 2(a)] with three of altogether seven similar measurements on $7 \mathrm{amu}$. As seen in Fig. 2(a) the formation of pure ${ }^{4} \mathrm{He}$ clusters beyond the tetramer is now largely suppressed. The mixed clusters on mass $7 \mathrm{amu}$ in parts (b)-(d) confirm the previous assignments of $(2,1),(3,1)$, and $(4,1)$. In addition, new peaks are seen at $(2,2)$ in Fig. 2(b) and at $(3,2)$ in Figs. 2(c) and 2(d). The $(4,2)$ peak is greatly enhanced at the expense of the $(5,1)$ peak. A fit of the next broad peak in Fig. 2(c) suggests contributions from $(6,1),(5,2)$, and $(4,3)$, which is indicated by the crosshatching. A fit of the same region in Fig. 2(d) confirms these assignments and suggests possibly a small peak between $(5,1)$ and $(4,3)$, which may be the metastable $(3,4)$ cluster (Table I).

Table I summarizes the observations, predicted binding energies and degeneracies of all the possible mixed clusters up to $31 \mathrm{amu}(7,1)$. Excited states with different angular momentum quantum numbers $(L, S)$ are also included.

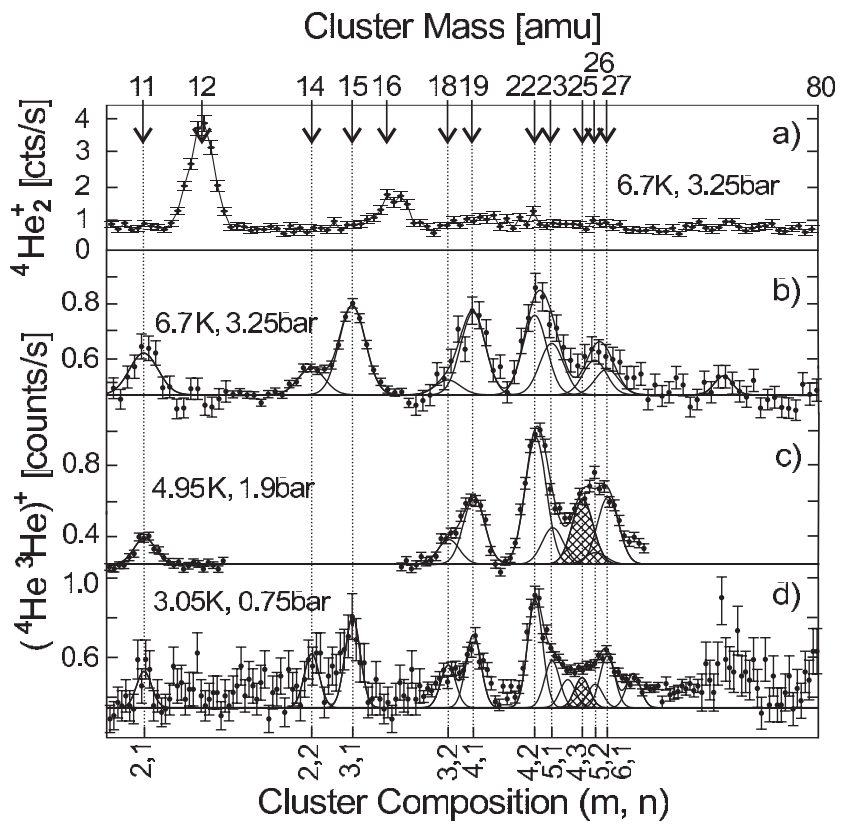

FIG. 2. Comparison of diffraction patterns measured with $1 \%$ ${ }^{4} \mathrm{He}$ in ${ }^{3} \mathrm{He}$ gas mixtures: (a) on mass $8 \mathrm{amu}\left({ }^{4} \mathrm{He}_{2}{ }^{+}\right)$and (b)(d) on mass 7 amu $\left({ }^{4} \mathrm{He}^{3} \mathrm{He}^{+}\right)$plotted on a mass scale (top abscissa) and composition scale (bottom abscissa). The smaller \pm standard deviations result from longer measurement times. The crosshatched region corresponds to the ${ }^{4} \mathrm{He}_{4}{ }^{3} \mathrm{He}_{3}$ cluster. The small peak in $(\mathrm{d})$ between $(5,1)$ and $(4,3)$ might correspond to the metastable ${ }^{4} \mathrm{He}_{3}{ }^{3} \mathrm{He}_{4}$ cluster. 
Clusters which do not form a bound state and clusters which form a metastable state are indicated "NB" and "MS," respectively. The predictions are used to discriminate peaks in cases in which two different $(m, n)$ assignments have the same mass. Thus the peak at mass $19 \mathrm{amu}$ is a $(4,1)$ and not a $(1,5)$ cluster since the latter is predicted to be unbound. But the peak at mass 27 amu may be due to either a $(6,1)$ or a $(3,5)$ cluster.

Some information on the relative stabilities can be extracted from the relative populations derived from the peak intensities. To predict free jet cluster populations a simple sudden freeze model was used. The model assumes that the cluster mole fractions are in equilibrium with the local temperature up to some point in the early stages of the expansion at which the equilibrium state is frozen in [15].

To implement the equilibrium model the experimental peak intensities were converted to mole fractions $x_{(m, n)}^{\text {exp } t}$ by the following formula [14]:

$$
x_{(m, n)}^{\exp t}=\frac{I(m, n)}{(m+n) f(m, n) I_{\mathrm{tot}}},
$$

where the total intensity $I_{\text {tot }}$ is assumed to be equal to the predominant ${ }^{3} \mathrm{He}$ atom peak intensity, and the factor $(m+$ $n$ ) corrects for the ionization probability of the cluster [16]. $f(m, n)$ is the probability that a $\left({ }^{4} \mathrm{He}^{3} \mathrm{He}\right)^{+}$fragment ion is formed upon ionization of a mixed cluster. If the probability for forming a $\left({ }^{4} \mathrm{He}^{3} \mathrm{He}\right)^{+}$ion or a ${ }^{3} \mathrm{He}_{2}{ }^{+}$ion is the same as for forming a ${ }^{4} \mathrm{He}_{2}{ }^{+}$ion in a pure ${ }^{4} \mathrm{He}_{N}$ cluster, then the following expression could be derived:

$$
f(m, n)=f^{\prime}(m+n) \frac{2 m n}{(m+n)(m+n-1)},
$$

where $f^{\prime}(3)=0.41, f^{\prime}(4)=0.68, f^{\prime}(\geq 5)=0.65$ [14], and the second term accounts for the number of combinations which can form a $\left({ }^{4} \mathrm{He}^{3} \mathrm{He}\right)^{+}$ion.

The mole fractions of the mixed clusters can be calculated from the partition functions for the equilibrium $m^{4} \mathrm{He}+n^{3} \mathrm{He} \leftrightarrow{ }^{4} \mathrm{He}_{m}{ }^{3} \mathrm{He}_{n}$ with the result
TABLE I. Comparison of observed peaks with theory predic-

\begin{tabular}{|c|c|c|c|c|}
\hline $\begin{array}{l}\text { Total mass } \\
\text { [amu] }\end{array}$ & Experiment $^{\mathrm{a}}$ & $(m, n)^{\mathrm{b}}$ & $\begin{array}{l}\text { Binding energy } \\
{[\mathrm{K}]^{\mathrm{c}}}\end{array}$ & $g^{\mathrm{d}}$ \\
\hline 11 & ++ & $(2,1)$ & 0.0142 & 2 \\
\hline 13 & 0 & $(1,3)$ & NB & $\ldots$ \\
\hline 14 & + & $(2,2)$ & 0.0997 & 1 \\
\hline 15 & ++ & $(3,1)$ & 0.297 & 2 \\
\hline 16 & 0 & $(1,4)$ & NB & $\ldots$ \\
\hline 17 & 0 & $(2,3)$ & $0.011 \mathrm{MS}$ & 6 \\
\hline 18 & + & $(3,2)$ & 0.574 & 1 \\
\hline \multirow[t]{2}{*}{19} & ++ & $(4,1)$ & 0.911 & 2 \\
\hline & & $(1,5)$ & NB & $\cdots$ \\
\hline 20 & 0 & $(2,4)$ & NB & $\ldots$ \\
\hline 21 & 0 & $(3,3)$ & $0.576 \mathrm{MS}$ & 6 \\
\hline \multirow[t]{3}{*}{22} & ++ & $(4,2)$ & 1.363 & 1 \\
\hline & & $(4,2)^{*}$ & 0.931 & 9 \\
\hline & & $(1,6)$ & NB & $\cdots$ \\
\hline \multirow[t]{2}{*}{23} & ++ & $(5,1)$ & 1.818 & 2 \\
\hline & & $(2,5)$ & NB & \\
\hline 24 & $?$ & $(3,4)$ & MS & 15 \\
\hline \multirow[t]{2}{*}{25} & + & $(4,3)$ & 1.464 & 6 \\
\hline & & $(1,7)$ & NB & $\cdots$ \\
\hline \multirow[t]{3}{*}{26} & + & $(5,2)$ & 2.427 & 1 \\
\hline & & $(5,2)^{*}$ & 1.968 & 9 \\
\hline & & $(2,6)$ & NB & $\ldots$ \\
\hline \multirow[t]{2}{*}{27} & ++ & $(6,1)$ & 2.980 & 2 \\
\hline & & $(3,5)$ & 0.757 & 20 \\
\hline \multirow[t]{2}{*}{28} & 0 & $(4,4)$ & 1.719 & 15 \\
\hline & & $(1,8)$ & NB & $\cdots$ \\
\hline \multirow[t]{2}{*}{29} & $?$ & $(5,3)$ & 2.660 & 6 \\
\hline & & $(2,7)$ & NB & $\cdots$ \\
\hline \multirow[t]{3}{*}{30} & $?$ & $(6,2)$ & 3.737 & 1 \\
\hline & & $(6,2)^{*}$ & 3.265 & 9 \\
\hline & & $(3,6)$ & 0.923 & 15 \\
\hline \multirow[t]{3}{*}{31} & ++ & $(7,1)$ & 4.365 & 2 \\
\hline & & $(4,5)$ & 2.011 & 20 \\
\hline & & $(1,9)$ & NB & $\cdots$ \\
\hline
\end{tabular}
tions of mixed clusters.

${ }^{\mathrm{a}} 0$, no signal; + , weak peak; ++ , distinct strong peak; ?, uncertain, peak possible but not sufficiently resolved.

${ }^{\mathrm{b}}$ An asterisk indicates an excited state.

${ }^{\mathrm{c}}$ All the binding energies are from Ref. [10] or, when not available there, from Ref. [9]. No bound states (NB) and metastable states (MS) were predicted in Ref. [9].

$\mathrm{d}_{g}=(2 L+1)(2 S+1)$ is the degeneracy of the state with total orbital angular momentum $L$ and total nuclear spin $S$.

$$
\begin{aligned}
x_{(m, n)}^{\mathrm{th}} & =\alpha^{m}(1-\alpha)^{n}\left(\frac{4 m+3 n}{4^{m} 3^{n}}\right)^{3 / 2}\left[\frac{P_{0}}{\left(k T_{0}\right)^{5 / 2}}\left(\frac{2 \pi \hbar^{2}}{M_{1}}\right)^{3 / 2}\right]^{m+n-1} \frac{1}{2^{n}} \sum_{j} g_{j}(m, n) \exp \left(\frac{E_{j}(m, n)}{k T_{\mathrm{SF}}(m, n)}\right), \\
& =X\left(m, n, \alpha, P_{0}, T_{0}\right) \sum_{j} g_{j}(m, n) \exp \left(\frac{E_{j}(m, n)}{k T_{\mathrm{SF}}(m, n)}\right),
\end{aligned}
$$

where $\alpha$ is the ${ }^{4} \mathrm{He}$ mole fraction in the source, $M_{1}=1 \mathrm{amu}, g_{j}(m, n)$ is the degeneracy of the cluster bound state $j$ with binding energy $E_{j}(m, n), T_{\mathrm{SF}}(m, n)$ is the cluster sudden freeze temperature, and $k$ is Boltzmann's constant. Figure 3 shows the sudden freeze temperatures $T_{\mathrm{SF}}(m, n)$ for clusters larger than $(2,1)$ [17] for the measurements in Figs. 1 and 2 [18]. It is gratifying to find that the temperatures follow, with few exceptions, a smooth trend increasing with the cluster mass. The best fit curves for the 9\% and $10 \%$ mixtures are only slightly different and those for the three $1 \%$ mixtures are almost completely identical. The sudden freeze temperatures increase with cluster size from about 15 up to $180 \mathrm{mK}$. This is 


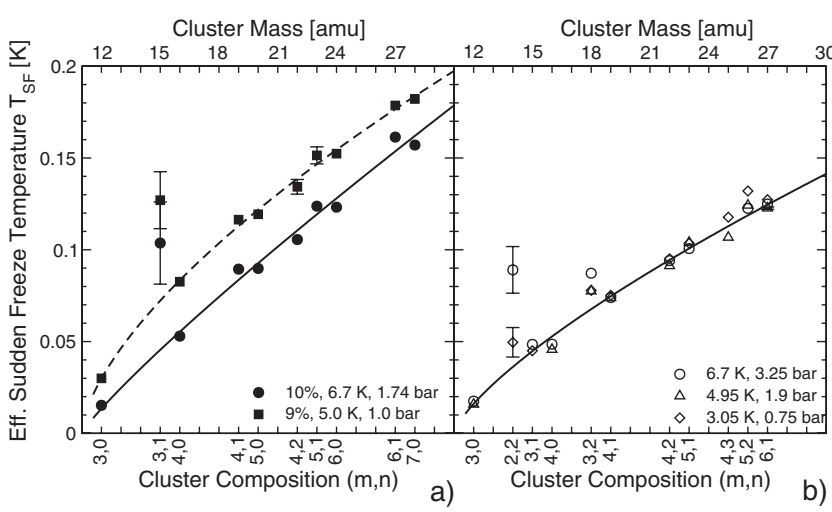

FIG. 3. Effective cluster formation sudden freeze temperatures evaluated with Eq. (3) for the measurements in Figs. 1 and 2. Major deviations from the otherwise smooth correlation are found only for the $(3,1)$ cluster in (a) and for $(2,2)$ in (b). With these exceptions, standard deviations are generally smaller than the size of the symbols.

expected since the large number of sequential collision processes needed to produce the large clusters is available only in the early hotter stages of the expansion. The smaller clusters, which require fewer collisions, continue to be in equilibrium farther downstream at lower temperatures.

The smooth progression of the sudden freeze temperatures indicates that most of the binding energies are consistent with the data. The only possible deviations are for $(2,2)$ in the $1 \%{ }^{3} \mathrm{He}$ mixtures and for $(3,1)$ in the $9 \%-10 \%$ experiments, where the predicted $T_{\mathrm{SF}}$ are too high. Since the $(3,1)$ cluster falls on the experimental curve in the $1 \%$ ${ }^{3} \mathrm{He}$ mixture, the observed deviation in this case may not be significant.

In summary, nondestructive cluster mass spectroscopy based on matter-wave diffraction has been used to detect a large number of ${ }^{4} \mathrm{He}_{m}{ }^{3} \mathrm{He}_{n}$ clusters with up to 8 atoms. An analysis of the relative experimental mole fraction suggests that the calculated binding energies for all clusters with the possible exception of the ${ }^{4} \mathrm{He}_{2}{ }^{3} \mathrm{He}_{2}$ are consistent with the measurements. The remarkable correlation between the effective sudden freeze temperatures and the cluster masses provides new support for a sudden freeze model of cluster growth.

In the future it should be possible to investigate the magnetic moments of the mixed clusters by deflecting them in an inhomogeneous Stern-Gerlach-type magnet as recently demonstrated for $\mathrm{H}_{2}$ clusters [19]. In this experiment, only clusters with nonzero total spin are expected to be deflected.

We are grateful to T. Savas and H. I. Smith for providing the gratings, which made these experiments possible. We also thank F. Buyvol-Kot for help with the calculations, which led to the sudden freeze model. We also profited from correspondence and discussions on the the- ory with D. Bressanini, L. Bruch, R. Guardiola, A. S. Jensen, M. Lewerenz, and J. Navarro.

*Corresponding author.

Electronic address: okornil@gwdg.de

${ }^{\dagger}$ Present address: Fritz-Haber-Institut der Max-PlanckGesellschaft, Abteilung Molekülphysik, Faradayweg 4-6, 14195 Berlin, Germany.

[1] See, for example, E. Krotscheck and R. Zillich, J. Chem. Phys. 115, 10161 (2001).

[2] R.E. Grisenti, W. Schöllkopf, J.P. Toennies, G. Hegerfeldt, T. Köhler, and M. Stoll, Phys. Rev. Lett. 85, 2284 (2000).

[3] R. Brühl, R. Guardiola, A. Kalinin, O. Kornilov, J. Navarro, T. Savas, and J. P. Toennies, Phys. Rev. Lett. 92, 185301 (2004).

[4] S. Weisgerber and P.-G. Reinhard, Z. Phys. D 23, 275 (1992); Y. Yannouleas and U. Landman, Phys. Rev. B 54, 7690 (1996).

[5] R. Guardiola and J. Navarro, Phys. Rev. A 71, 035201 (2005).

[6] S. Nakaichi, T. K. Lim, Y. Akaishi, and H. Tanaka, J. Chem. Phys. 71, 4430 (1979).

[7] E. Nielsen, D. V. Fedorov, and A. S. Jensen, J. Phys. B 31, 4085 (1998).

[8] D. Bressanini, M. Zavaglia, M. Mella, and G. Morosi, J. Chem. Phys. 112, 717 (2000); D. Bressanini and G. Morosi, Phys. Rev. Lett. 90, 133401 (2003).

[9] R. Guardiola and J. Navarro, Phys. Rev. A 68, 055201 (2003).

[10] D. Bressanini and G. Morosi, Few-Body Syst. 34, 131 (2004).

[11] A. S. Jensen, K. Riisager, D. V. Fedorov, and E. Garrido, Rev. Mod. Phys. 76, 215 (2004).

[12] M. Lewerenz, J. Chem. Phys. 106, 4596 (1997).

[13] R. E. Grisenti, W. Schöllkopf, J. P. Toennies, J. R. Manson, T. A. Savas, and H. I. Smith, Phys. Rev. A 61, 033608 (2000).

[14] L. W. Bruch, W. Schöllkopf, and J. P. Toennies, J. Chem. Phys. 117, 1544 (2002).

[15] D. R. Miller, in Atomic and Molecular Beam Methods, edited by G. Scoles (Oxford University Press, Oxford, 1988), Vol. 1, Chap. 2.

[16] The ionization cross sections for ${ }^{4} \mathrm{He}$ and ${ }^{3} \mathrm{He}$ atoms are assumed to be equal.

[17] This method breaks down for the $(2,1)$ cluster since its binding energy $(0.0142 \mathrm{~K})$ is almost the same in absolute value as the translational sudden freeze temperature, which for these ${ }^{3} \mathrm{He}$ rich mixtures is estimated to be between 0.006 and $0.010 \mathrm{~K}$.

[18] The binding energies of the pure ${ }^{4} \mathrm{He}$ clusters are from Ref. [12]: (3, 0), 0.126 K; (4, 0), $0.558 \mathrm{~K} ;(5,0), 1.2999 \mathrm{~K}$; $(6,0), 2.313 \mathrm{~K} ;(7,0), 3.568 \mathrm{~K}$.

[19] F. Buyvol-Kot, A. Kalinin, O. Kornilov, J. P. Toennies, and J. A. Becker, Solid State Commun. 135, 532 (2005). 